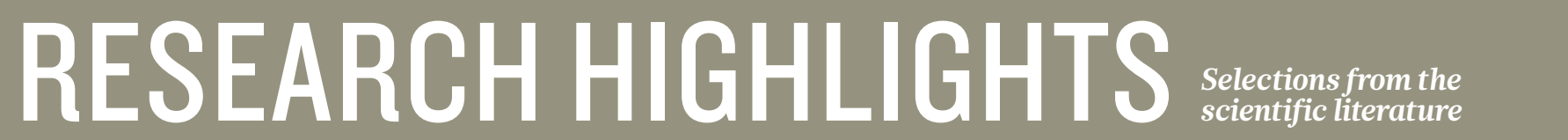

GENETIC ENGINEERING

\section{Boosting 'gene drive' safety}

Researchers have developed a way to reduce the risks of a method that genetically engineers entire populations with unprecedented speed.

'Gene drives' are genetic changes, based on inserting parts of the CRISPR-Cas9 genome-editing system into a host genome, that spread through a population more rapidly than do normal mutations. Gene drives could be used to wipe out disease-carrying insects, for example, but could also spread uncontrollably in an ecosystem.

To reduce this risk, Kevin Esvelt and George Church of Harvard Medical School in Boston, Massachusetts, and their team inserted the bacterial DNA-cutting Cas9 enzyme into a piece of DNA external to the Saccharomyces cerevisiae yeast genome, and put the guide RNAs for directing Cas9 to a specific DNA sequence into the genome. This separation ensured that the gene drive would not spread exponentially if the strain was released into the wild.

Nature Biotech. http://doi. org/89h (2015)

ANIMAL BEHAVIOUR

\section{Polarized light as a secret signal}

Some crustaceans can detect polarized light, using it as a covert signal that is

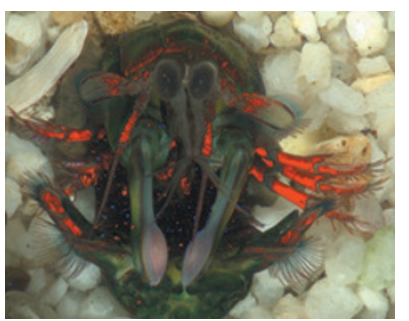

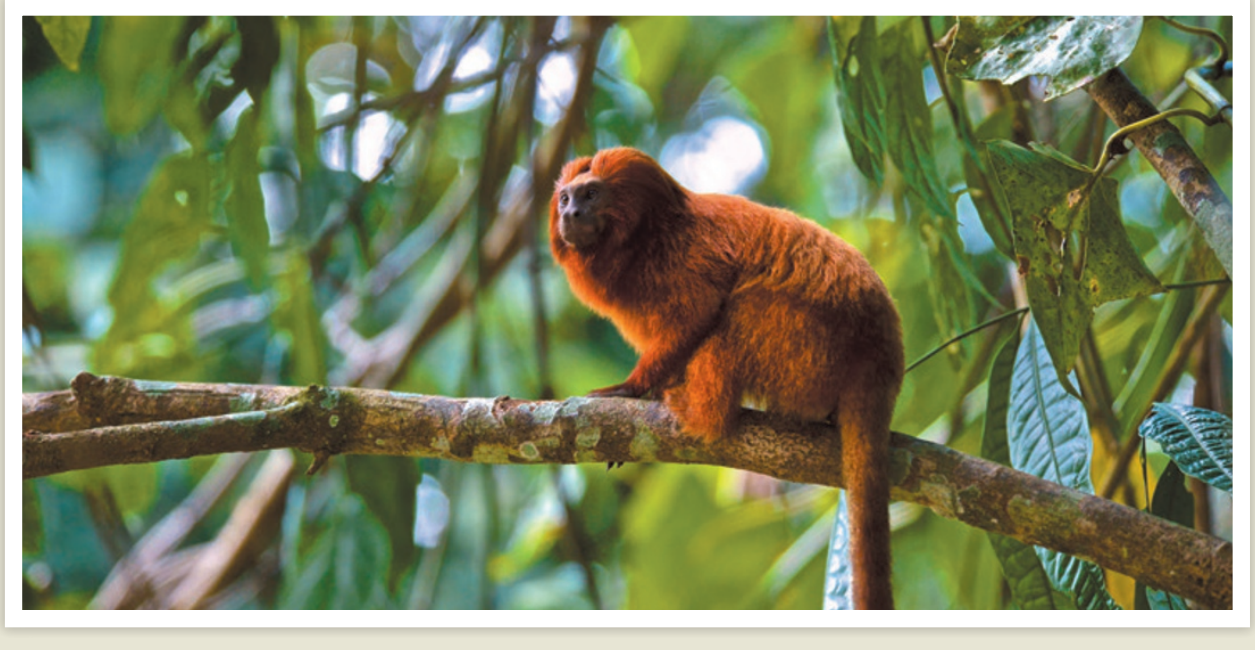

CONSERVATION

\section{How to save a species}

Common factors such as law enforcement contribute to the success or failure of speciesrecovery programmes, suggesting that conservation lessons could be generalized across different populations or species.

Jennifer Crees at the Zoological Society of London and her colleagues analysed 48 mammalian conservation programmes, ranging from the successful protection of the golden lion tamarin (pictured) to the failed attempt to save the Yangtze River dolphin.
Whereas national-level legislation did not necessarily lead to good outcomes, intensity of local law enforcement did. Moreover, reducing the threats to animals - such as habitat loss and hunting - was crucial for long-term survival.

No link was found between the outcomes of recovery programmes and biological factors such as body mass and habitat type, suggesting that well-designed conservation programmes should work across different species. Conserv. Biol. http://doi.org/87v (2015) invisible to predators.

Yakir Luc Gagnon at the University of Queensland in Brisbane, Australia, found that the bodies of mantis shrimps (Gonodactylaceus falcatus; pictured) reflect a distinctive pattern of circular polarization (pictured in red) that is visible only to other shrimps. When presented with different burrows in the laboratory, mantis shrimps avoided or delayed entering those that were lit with circularly polarized light compared with those under unpolarized light. This suggests that the shrimps use polarized light cues to sense whether potential burrows are occupied.

In another study, Martin
How at the University of Bristol, UK, found that male fiddler crabs ( $U c a$ stenodactylus) detected polarized targets in the wild from farther away than nonpolarized ones. The animals' sensitivity to polarized light could be boosting the visual contrast between crabs and their mudflat habitat.

Curr. Biol. http://doi.org/89c (2015); http://doi.org/89d (2015)

\section{MICROBIOLOGY \\ Antibiotics make MRSA worse}

Antibiotics could help a drug-resistant pathogen to worsen inflammation.

\section{Methicillin-resistant} Staphylococcus aureus (MRSA) resists most $\beta$-lactam antibiotics by acquiring a protein that modifies the cell wall. David Underhill, George Liu and their team at CedarsSinai Medical Center in Los Angeles, California, thought that this modification might also boost the production of inflammatory molecules called cytokines in the host. They exposed mouse and human immune cells to MRSA and found that the host cells made higher levels of a cytokine called IL- $1 \beta$ when MRSA had been grown in the presence of $\beta$-lactams. In mice, treatment with a $\beta$-lactam caused more immune cells to flood the site 